\title{
An Analysis of a Uniformly Convergent Finite Difference/Finite Element Scheme for a Model Singular-Perturbation Problem*
}

\author{
By Eugene C. Gartland, Jr.**
}

\begin{abstract}
Uniform $\mathscr{O}\left(h^{2}\right)$ convergence is proved for the El-Mistikawy-Werle discretization of the problem $-\varepsilon u^{\prime \prime}+a u^{\prime}+b u=f$ on $(0,1), u(0)=A, u(1)=B$, subject only to the conditions $a, b, f \in \mathscr{W}^{2, \infty}[0,1]$ and $a(x)>0,0 \leq x \leq 1$. The principal tools used are a certain representation result for the solutions of such problems that is due to the author [Math. Comp., v. 48, 1987, pp. 551-564] and the general stability results of Niederdrenk and Yserentant [Numer. Math., v. 41, 1983, pp. 223-253]. Global uniform $\mathscr{O}(h)$ convergence is proved under slightly weaker assumptions for an equivalent Petrov-Galerkin formulation.
\end{abstract}

1. Introduction. Consider the model singular-perturbation problem

$$
\begin{gathered}
L_{\varepsilon} u:=-\varepsilon u^{\prime \prime}+a u^{\prime}+b u=f \quad \text { on }(0,1), \\
u(0)=A, \quad u(1)=B .
\end{gathered}
$$

Here we assume that $\varepsilon$ is positive and that the coefficients, $a$ and $b$, and source term, $f$, satisfy

$$
\begin{aligned}
& \text { (a) } a, b, f \in \mathscr{W}^{1, \infty}, \\
& \text { (b) } \underline{a}:=\min \{a(x): 0 \leq x \leq 1\}>0,
\end{aligned}
$$

where $\mathscr{W}^{m, p}=\mathscr{W}^{m, p}[0,1]$ is the usual notation for the Sobolev space of functions on $[0,1]$ whose $m$ th derivatives are in $L^{p}$. Under these conditions, the problem (1.1) is well posed for all $\varepsilon$ sufficiently small, and it possesses a regular $\mathscr{O}(\varepsilon)$ boundary layer at the "downstream" end, $x=1$. In particular, we have the following.

Let $\||\cdot|\|_{\varepsilon}$ denote the weighted Sobolev norm

$$
\||v|\|_{\varepsilon}:=\max \left\{\|v\|_{\infty}, \varepsilon\left\|v^{\prime}\right\|_{\infty}\right\} \text {. }
$$

THEOREM 1.1. Under assumptions (1.2), there exist positive constants $\varepsilon_{0}$ and $c$ such that for all $v$ in $\mathscr{W}^{2,1}$

$$
\|v\|_{\varepsilon} \leq c\left\{\left\|L_{\varepsilon} v\right\|_{1}+|v(0)|+|v(1)|\right\}, \quad 0<\varepsilon \leq \varepsilon_{0} .
$$

This theorem is proved under the more general assumption of bounded coefficient functions in [4]. Versions of this result for general $m$ th-order linear problems with

Received April 30, 1986; revised April 27, 1987.

1980 Mathematics Subject Classification (1985 Revision). Primary 65L10, 65L60; Secondary $34 \mathrm{E} 15$.

*This work was supported in part by the National Science Foundation under grant DMS8602199.

${ }^{* *}$ Current address. Department of Mathematical Sciences, Kent State University, Kent, Ohio 44242.

(C) 1988 American Mathematical Society $0025-5718 / 88 \$ 1.00+\$ .25$ per page 
continuous coefficients are in [11]. It also follows from results in [14] and [15, §9.3]. A consequence of Theorem 1.1 is the strong uniform stability of "nearby" problems. Consider the perturbed differential operator

$$
\tilde{L}_{\varepsilon} v:=-\varepsilon v^{\prime \prime}+\tilde{a} v^{\prime}+\tilde{b} v,
$$

where we assume that $\tilde{a} \in L^{\infty}$ and $\tilde{b} \in L^{1}$.

THEOREM 1.2. If the discrepancies $\|a-\tilde{a}\|_{\infty}$ and $\|b-\tilde{b}\|_{1}$ between the coefficient functions of the operators $L_{\varepsilon}$ and $\tilde{L}_{\varepsilon}$ are sufficiently small (uniformly in $\varepsilon$ ), then there exists a positive constant $\tilde{c}$ such that for all $v$ in $\mathscr{W}^{2,1}$

$$
\|v\|_{\varepsilon} \leq \tilde{c}\left\{\left\|\tilde{L}_{\varepsilon} v\right\|_{1}+|v(0)|+|v(1)|\right\}, \quad 0<\varepsilon \leq \varepsilon_{0} .
$$

Proof. We first observe that the $L^{1}$-norm of the derivative of the solution of $L_{\varepsilon} u=f$ can be bounded in terms of $\|f\|_{1},|u(0)|$, and $|u(1)|:$ If $L_{\varepsilon} u=f$ then

$$
-\varepsilon u^{\prime \prime}+a u^{\prime}=f-b u
$$

and

$$
u^{\prime}(x)=u^{\prime}(1) e^{-(1 / \varepsilon) \int_{x}^{1} a}+\frac{1}{\varepsilon} \int_{x}^{1} e^{-(1 / \varepsilon) \int_{x}^{t} a}(f(t)-b(t) u(t)) d t
$$

so that

$$
\begin{aligned}
\left\|u^{\prime}\right\|_{1} & \leq\left|u^{\prime}(1)\right| \cdot \int_{0}^{1} e^{-(1 / \varepsilon) \int_{x}^{1} a}+\|f-b u\|_{1} \cdot \frac{1}{\varepsilon} \sup _{0<t<1} \int_{0}^{1} e^{-(1 / \varepsilon) \int_{x}^{t} a} d x \\
& \leq\left\|u^{\prime}\right\|_{\infty} \cdot \frac{\varepsilon}{a}+\left(\|f\|_{1}+\|b\|_{1} \cdot\|u\|_{\infty}\right) \cdot \frac{1}{a} \\
& \leq \frac{1}{a}\left(1+\|b\|_{1}\right) \cdot\|u\|_{\varepsilon}+\frac{1}{\underline{a}}\|f\|_{1} \\
& \leq c_{1}\left\{\|f\|_{1}+|u(0)|+|u(1)|\right\}, \quad 0<\varepsilon<\varepsilon_{0}
\end{aligned}
$$

where $c_{1}$ depends on $\underline{a},\|b\|_{1}$, and $c$ from (1.3). Next define

$$
\delta:=\max \left\{\|a-\tilde{a}\|_{\infty},\|b-\tilde{b}\|_{1}\right\}
$$

and bound $\left\|L_{\varepsilon} v\right\|_{1}$ in terms of $\left\|\tilde{L}_{\varepsilon} v\right\|_{1},|v(0)|$, and $|v(1)|$ (for $\delta$ sufficiently small):

$$
\begin{aligned}
\left\|L_{\varepsilon} v\right\|_{1} & \leq\left\|\tilde{L}_{\varepsilon} v\right\|_{1}+\|a-\tilde{a}\|_{\infty} \cdot\left\|v^{\prime}\right\|_{1}+\|b-\tilde{b}\|_{1} \cdot\|v\|_{\infty} \\
& \leq\left\|\tilde{L}_{\varepsilon} v\right\|_{1}+\|a-\tilde{a}\|_{\infty} \cdot c_{1} \cdot\left\{\left\|L_{\varepsilon} v\right\|_{1}+|v(0)|+|v(1)|\right\}+\|b-\tilde{b}\|_{1} \cdot\|v\| \|_{\varepsilon} \\
& \leq\left\|\tilde{L}_{\varepsilon} v\right\|_{1}+\mathscr{O}(\delta)\left\|L_{\varepsilon} v\right\|_{1}+\mathscr{O}(\delta)\{|v(0)|+|v(1)|\},
\end{aligned}
$$

that is,

$$
\left\|L_{\varepsilon} v\right\|_{1} \leq(1+\mathscr{O}(\delta))\left\|\tilde{L}_{\varepsilon} v\right\|_{1}+\mathscr{O}(\delta)\{|v(0)|+|v(1)|\} .
$$

Our result follows by combining the above inequality with the original stability property (1.3).

We note that the optimal stability constant above satisfies $\tilde{c}=c(1+\mathscr{O}(\delta))$ (if $c$ is the optimal constant in (1.3)). An immediate consequence of Theorem 1.2 is that to have a well-posed problem of the form (1.1), it is not necessary that the coefficient function $b(x)$ be bounded from below; it is enough for the $L^{1}$-norm of its negative part to be sufficiently small. We have the following, for example. 
COROLLARY 1.3. The differential operator $L_{\varepsilon}$ of (1.1) is strongly uniformly stable, in the sense of (1.3), for $0<\varepsilon<\infty$, if the $L^{1}$-norm of the negative part of $b(x)$ is sufficiently small.

Proof. Let $b^{+}$and $b^{-}$denote the positive part and negative part of $b$ :

$$
b^{+}(x)=\left\{\begin{array}{ll}
0, & \text { if } b(x) \leq 0, \\
b(x), & \text { if } b(x)>0,
\end{array} \quad b^{-}(x)= \begin{cases}b(x), & \text { if } b(x)<0 \\
0, & \text { if } b(x) \geq 0\end{cases}\right.
$$

The differential operator $L_{\varepsilon}^{+}$, defined by

$$
L_{\varepsilon}^{+} v:=-\varepsilon v^{\prime}+a v^{\prime}+b^{+} v
$$

satisfies the stability inequality

$$
\||| v\|_{\varepsilon} \leq c^{+}\left\{\left\|L_{\varepsilon}^{+} v\right\|_{1}+|v(0)|+|v(1)|\right\}, \quad 0<\varepsilon<\infty,
$$

for all sufficiently smooth $v$, where $c^{+}$is a constant that does not depend on $\varepsilon$-it can be shown directly that $L_{\varepsilon}^{+}$satisfies a maximum principle for $\varepsilon>0$ (see, for example, [17]). The desired result follows from Theorem 1.2 and the observation that $\left\|b^{+}-b\right\|_{1}=\left\|b^{-}\right\|_{1}$.

The analysis of discretizations of (1.1) (or any differential operator, for that matter) in the case of unbounded coefficient functions is a difficult matter. Here we will be content to consider the case where $a, b$, and $f$ are in $\mathscr{W}^{1, \infty}$, for our global error estimate, and in $\mathscr{W}^{2, \infty}$, later. In this case (since $a$ and $b$ are uniformly continuous on $[0,1])$, there will exist an $\varepsilon_{1}>0$ sufficiently small so that

$$
a^{2}(x)+4 \varepsilon b(x) \geq 0, \quad 0 \leq x \leq 1,0<\varepsilon \leq \varepsilon_{1} .
$$

If the maximum possible values for $\varepsilon_{1}$ and/or $\varepsilon_{0}$ are $+\infty$, then we will take them to be some arbitrarily prescribed finite values; the problem (1.1) can be analyzed by classical techniques for $\varepsilon$ large. Some of the analysis that follows will then be broken up into the ranges $0<\varepsilon \leq \varepsilon_{1}$, on which $a^{2}+4 \varepsilon b \geq 0$ is assured, and $\varepsilon_{1} \leq \varepsilon \leq \varepsilon_{0}$, on which $u$ and its derivatives can be bounded independently of $\varepsilon$.

We are interested in the error analysis of a well-known difference approximation to (1.1) proposed by El-Mistikawy and Werle in [3]. Let a uniform partition of the interval $[0,1]$ be given by $x_{i}=i h, i=0, \ldots, n$, where $h=1 / n$. Let $u^{h}$ denote a discrete approximation on this mesh to the solution $u$ of (1.1), and let $g_{i}$ denote $g\left(x_{i}\right)$ for any function $g$ defined on the mesh. The El-Mistikawy scheme is of the form

$$
\begin{gathered}
L_{h, \varepsilon} u_{i}^{h}:=\alpha_{i,-1} u_{i-1}^{h}+\alpha_{i, 0} u_{i}^{h}+\alpha_{i, 1} u_{i+1}^{h} \\
=\beta_{i,-1} f_{i-1}+\beta_{i, 0} f_{i}+\beta_{i, 1} f_{i+1}=: I_{h}(f)_{i}, \quad i=1, \ldots n-1, \\
u_{0}^{h}=A, \quad u_{n}^{h}=B .
\end{gathered}
$$

The expressions for the coefficients and weights are rather complicated; they are characterized in the next section.

A finite difference approximation to (1.1) is said to be uniformly accurate of order $p$ (with respect to some norm $\|\cdot\|$ ) if the associated discretization error, $e^{h}=u-u^{h}$, satisfies $\left\|e^{h}\right\| \leq c h^{p}$ for all $0<h \leq h_{0}$ and $0<\varepsilon \leq \varepsilon_{0}$, with an asymptotic error constant $c$ that does not depend on $\varepsilon$. The El-Mistikawy-Werle scheme is uniformly $\mathscr{O}\left(h^{2}\right)$. This has been shown in the case $b(x) \equiv 0$ by Berger, Solomon, and Ciment 
in [1], Hegarty, Miller, and O'Riordan in [7], and O'Riordan and Stynes in [12]. It has been established in the case $b(x) \geq 0, a(x) \equiv$ const $>0$ by Lorenz in [10] and in the case $b(x) \geq \underline{b}, \underline{a}^{2}+4 \varepsilon \underline{b}>0$ by Stynes and O'Riordan in [16].

It is the purpose of this note to show how tools developed in [4] and [5] for the analysis of a family of uniformly accurate discretizations of (1.1) (known as exponentially fitted HODIE schemes) can be used to prove the uniform $\mathscr{O}\left(h^{2}\right)$ convergence of the El-Mistikawy-Werle scheme. The analysis does not require the previously needed conditions on the coefficient function $b$; it is simpler than the above cited papers; it requires less smoothness of the data; and it gives convergence with respect to stronger norms. In addition, as a preliminary step, global uniform $\mathscr{O}(h)$ convergence results, which generalize earlier work, are proved using stability/perturbation results.

2. The El-Mistikawy-Werle Scheme. The El-Mistikawy-Werle scheme is of the form (1.4); it can be derived in various ways. It was first proposed in [3], where it was derived by approximating the coefficient functions $a$ and $b$ and source term $f$ in (1.1) by piecewise constant functions

$$
\bar{a}(x)=\bar{a}_{i}=\frac{a_{i}+a_{i+1}}{2}, \quad \bar{b}(x)=\bar{b}_{i}=\frac{b_{i}+b_{i+1}}{2},
$$

and

$$
\bar{f}(x)=\bar{f}_{i}=\frac{f_{i}+f_{i+1}}{2}, \quad x_{i}<x<x_{i+1}, i=0, \ldots, n-1,
$$

and then constructing a three-point scheme that is satisfied identically by the solution of the approximate problem. It was shown in [1] that this scheme results when one solves the approximate problem on $\left(x_{i-1}, x_{i}\right)$ and $\left(x_{i}, x_{i+1}\right)$ and requires that the solution be in $C^{1}\left(x_{i-1}, x_{i+1}\right)$. It can also be derived from Marchuk-type local integral relations by using two-point quadrature rules (see [9]). In [12] and [16], use was made of the fact that the El-Mistikawy-Werle scheme is equivalent to a discrete Petrov-Galerkin scheme. This point of view works to simplify many of our calculations below, and we describe it now.

Let $B_{\varepsilon}(\cdot, \cdot)$ and $\bar{B}_{\varepsilon}(\cdot, \cdot)$ denote the bilinear forms

$$
B_{\varepsilon}(v, w):=\int_{0}^{1}\left(\varepsilon v^{\prime} \omega^{\prime}+a v^{\prime} w+b v w\right)
$$

and

$$
\bar{B}_{\varepsilon}(v, w):=\int_{0}^{1}\left(\varepsilon v^{\prime} w^{\prime}+\bar{a} v^{\prime} w+\bar{b} v w\right)
$$

and let $(\cdot, \cdot)$ denote the usual $L^{2}$ inner product. Let $\left\{\phi_{0}, \ldots, \phi_{n}\right\}$ be any basis of trial functions in $\mathscr{W}^{1,2}$ that satisfy

$$
\begin{aligned}
& \operatorname{supp}\left(\phi_{i}\right) \subseteq\left[x_{i-1}, x_{i+1}\right], \\
& \phi_{i}\left(x_{j}\right)=\delta_{i j}, \quad i, j=0, \ldots, n .
\end{aligned}
$$

And let $\left\{\psi_{1}, \ldots, \psi_{n-1}\right\}$ be the basis of local-support test functions given by

$$
\begin{aligned}
& \operatorname{supp}\left(\psi_{i}\right) \subseteq\left[x_{i-1}, x_{i+1}\right] \\
& -\varepsilon \psi_{i}^{\prime \prime}-\bar{a} \psi_{i}^{\prime}+\bar{b} \psi_{i}=0 \quad \text { on }\left(x_{i-1}, x_{i}\right) \cup\left(x_{i}, x_{i+1}\right) \\
& \psi_{i}\left(x_{i-1}\right)=\psi_{i}\left(x_{i+1}\right)=0, \quad \psi_{i}\left(x_{i}\right)=1
\end{aligned}
$$


Then the coefficients and weights in the finite difference scheme (1.4) are given by

$$
\begin{aligned}
& \alpha_{i,-1}=\frac{1}{h} \bar{B}_{\varepsilon}\left(\phi_{i-1}, \psi_{i}\right), \quad \alpha_{i, 0}=\frac{1}{h} \bar{B}_{\varepsilon}\left(\phi_{i}, \psi_{i}\right), \quad \alpha_{i, 1}=\frac{1}{h} \bar{B}_{\varepsilon}\left(\phi_{i+1}, \psi_{i}\right), \\
& \beta_{i,-1}=\frac{1}{2 h} \int_{x_{i-1}}^{x_{i}} \psi_{i}, \quad \beta_{i, 0}=\frac{1}{2 h} \int_{x_{i-1}}^{x_{i+1}} \psi_{i}, \quad \beta_{i, 1}=\frac{1}{2 h} \int_{x_{i}}^{x_{i+1}} \psi_{i},
\end{aligned}
$$

and the resulting linear algebraic system is identical (after scaling by $h$ ) to that associated with the Petrov-Galerkin approximation

$$
\begin{aligned}
& u^{h}=\sum_{i=0}^{n} u_{i}^{h} \phi_{i}, \\
& \bar{B}_{\varepsilon}\left(u^{h}, \psi_{i}\right)=\left(\bar{f}, \psi_{i}\right), \quad i=1, \ldots, n-1, \\
& u^{h}(0)=A, \quad u^{h}(1)=B .
\end{aligned}
$$

Explicit formulas for the $\alpha$ 's and $\beta$ 's in (2.1) are given in [1] for the case where $\bar{a}^{2}+4 \varepsilon \bar{b} \geq 0$. We observe that this procedure is well defined, even when this condition is not met, provided $h$ is sufficiently small.

PROPOSITION 2.1. Under assumptions (1.2), the finite difference scheme $(1.4) /(2.1)$ is well defined for all $0<\varepsilon \leq \varepsilon_{0}$ and $0<h \leq h_{0}$ for some sufficiently small $h_{0}$ that does not depend on $\varepsilon$.

Proof. We only need to know that the local problems defining the $\psi_{i}$ functions are all well posed for $h$ sufficiently small. Let $\bar{L}_{\varepsilon}$ denote the differential operator associated with the piecewise constant functions $\bar{a}$ and $\bar{b}$ :

$$
\bar{L}_{\varepsilon} v:=-\varepsilon v^{\prime \prime}+\bar{a} v^{\prime}+\bar{b} v .
$$

Now, by the assumed smoothness of $a$ and $b$ and the construction of $\bar{a}$ and $\bar{b}$, it follows that $\|a-\bar{a}\|_{\infty}$ and $\|b-\bar{b}\|_{1}$ are both $\mathscr{O}(h)$. So Theorem 1.2 implies that the operator $\bar{L}_{\varepsilon}$ is strongly uniformly stable, in the sense (1.3), for all $0<\varepsilon \leq \varepsilon_{0}$ and $0<h \leq h_{0}$ for some $h_{0}$.

Next we observe that for this range of $\varepsilon$ and $h$, all of the eigenvalues of $\bar{L}_{\varepsilon}$ must be positive. To see this, we first note that the eigenvalue problem for $L_{\varepsilon}$ (namely $-\varepsilon \phi^{\prime \prime}+a \phi^{\prime}+b \phi=\lambda \phi, \phi(0)=\phi(1)=0$ ) can be symmetrized by the Sturm Transformation $\phi=\psi \exp \left(\int a / 2 \varepsilon\right)$ to yield the variational characterization

$$
\lambda_{\min }=\inf _{\phi \in H_{0}^{1}} \frac{\int_{0}^{1} \varepsilon\left(\phi^{\prime}\right)^{2}+\left(a^{2} / 4 \varepsilon+b-a^{\prime} / 2\right) \phi^{2}}{\int_{0}^{1} \phi^{2}} .
$$

From this it follows that $\lambda_{\min } \rightarrow+\infty$ as $\varepsilon \rightarrow 0$.

Now for each $\varepsilon>0$, the differential operator $L_{\varepsilon}$ is a closed operator on $\mathscr{W}_{\varepsilon}^{1, \infty}$ (the Sobolev space $\mathscr{W}^{1, \infty}$ normed by $\left.\|\mid \cdot\| \|_{\varepsilon}\right)$ to $L^{1}$ with domain $D\left(L_{\varepsilon}\right)=\mathscr{W}_{0}^{2,1}$. It follows from the estimates in the proof of Theorem 1.2 that for $v \in D\left(L_{\varepsilon}\right)$

$$
\left\|\left(L_{\varepsilon}-\bar{L}_{\varepsilon}\right) v\right\|_{1} \leq \mathscr{O}(h)\left\{\|\| v\|\|_{\varepsilon}+\left\|L_{\varepsilon} v\right\|_{1}\right\} .
$$

So $\bar{L}_{\varepsilon}$ converges to $L_{\varepsilon}$ in the sense of generalized convergence ([8, Chapter IV, §2.6]), uniformly in $\varepsilon$ as $h \rightarrow 0$. Thus $\bar{\lambda}_{\text {min }}$, the minimum eigenvalue of $\bar{L}_{\varepsilon}$, must converge to $\lambda_{\min }$ as $h \rightarrow 0$ (uniformly in $\varepsilon$ ); see [8, Chapter IV, §3.5]. 
Therefore, $\bar{\lambda}_{\min }$ is positive for all $\varepsilon$ and $h$ sufficiently small. This conclusion can be extended to the whole range $0<\varepsilon \leq \varepsilon_{0}$ and $0<h \leq h_{0}$ by continuity: for $\bar{\lambda}_{\text {min }}$ to vanish anywhere in this range would violate the assumed stability of $\bar{L}_{\varepsilon}$.

Finally, positivity of the eigenvalues assures us that $\bar{L}_{\varepsilon}$ possesses a nonnegative Green's function and a minimum principle. So there can be no conjugate points in $[0,1]$, i.e., for any $0 \leq a<b \leq 1$,

$$
\bar{L}_{\varepsilon} v=0 \quad \text { on }(a, b), \quad v(a)=v(b)=0
$$

implies $v \equiv 0$. See, for example, [13]. Thus the well-posedness of the local problems defining the $\psi_{i}$ functions in the El-Mistikawy-Werle scheme is guaranteed.

We remark that we had to resort to stability results for eigenvalues because there does not seem to be an easy way to symmetrize the differential operator $\bar{L}_{\varepsilon}$, whose coefficient function $\bar{a}$ is not smooth. We already at this stage have enough information to establish that the El-Mistikawy-Werle scheme is uniformly $\mathscr{O}(h)$, both nodally and globally, in its Petrov-Galerkin formulation, for certain types of trial functions.

THEOREM 2.2. Let the trial functions $\left\{\phi_{0}, \ldots, \phi_{n}\right\}$ satisfy for $0<\varepsilon \leq \varepsilon_{0}$ and $0<h \leq h_{0}$

$$
\int_{x_{i}}^{x_{i+1}}\left|\bar{L}_{\varepsilon} \phi_{j}\right| \leq c \cdot h, \quad i=0, \ldots, n-1, j=i, i+1,
$$

where $c$ is a constant that does not depend on $\varepsilon$ or $h$. Then the error, $e^{h}=u-u^{h}$, in the approximation (2.2) to (1.1) satisfies

$$
\left\|e^{h}\right\|_{\varepsilon} \leq c^{\prime} \cdot h, \quad 0<\varepsilon \leq \varepsilon_{0}, 0<h \leq h_{0},
$$

where $c^{\prime}$ does not depend on $\varepsilon$ or $h$.

Proof. Let $\bar{u}$ denote the true solution of the piecewise constant perturbed problem

$$
\bar{L}_{\varepsilon} \bar{u}=\bar{f}, \quad \bar{u}(0)=A, \quad \bar{u}(1)=B .
$$

Now, $\|u-\bar{u}\|_{\varepsilon}$ is uniformly $\mathscr{O}(h)$ : This follows from

$$
\begin{gathered}
L_{\varepsilon}(u-\bar{u})=(\bar{a}-a) u^{\prime}+(\bar{b}-b) u+(\bar{f}-f), \\
(u-\bar{u})(0)=(u-\bar{u})(1)=0
\end{gathered}
$$

plus the strong uniform stability of $L_{\varepsilon}$ and previously established estimates on $\|\bar{a}-a\|_{\infty},\left\|u^{\prime}\right\|_{1},\|\bar{b}-b\|_{1},\|u\|_{\infty}$, and $\|\bar{f}-f\|_{1}$.

Let $\bar{g}(x, y ; \varepsilon)$ denote the Green's function for $\bar{L}_{\varepsilon}$. Then $\bar{g}$ is well defined for $0<\varepsilon \leq \varepsilon_{0}$ and $0<h \leq h_{0}$, and it satisfies $\bar{B}_{\varepsilon}(v, \bar{g}(x, \cdot ; \varepsilon))=v(x)$ for all $v$ in $\mathscr{W}_{0}^{1,1}$. Moreover, for each nodal value $x_{i}, \bar{g}\left(x_{i}, \cdot ; \varepsilon\right) \in \operatorname{span}\left\{\psi_{1}, \ldots, \psi_{n-1}\right\}$. From this we can deduce that the Petrov-Galerkin scheme (2.2) is well posed:

$$
\bar{B}_{\varepsilon}\left(v^{h}, \psi_{i}\right)=0, \quad i=1, \ldots, n-1, \quad v^{h}(0)=v^{h}(1)=0
$$

implies

$$
v^{h}\left(x_{i}\right)=0, \quad i=0, \ldots, n,
$$

from which it follows that

$$
v^{h} \equiv 0
$$


It also follows that $u^{h}$ agrees exactly with $\bar{u}$ at the nodes:

$$
\begin{aligned}
\left(\bar{u}-u^{h}\right)\left(x_{i}\right) & =\bar{B}_{\varepsilon}\left(\bar{u}-u^{h}, \bar{g}\left(x_{i}, \cdot ; \varepsilon\right)\right) \\
& =\sum_{j=1}^{n-1} \bar{g}\left(x_{i}, x_{j} ; \varepsilon\right) \bar{B}_{\varepsilon}\left(\bar{u}-u^{h}, \psi_{j}\right) \\
& =\sum_{j=1}^{n-1} \bar{g}\left(x_{i}, x_{j} ; \varepsilon\right)\left[\left(\bar{f}, \psi_{j}\right)-\bar{B}_{\varepsilon}\left(u^{h}, \psi_{j}\right)\right]=0 .
\end{aligned}
$$

Now on each subinterval the difference $\bar{u}-u^{h}$ satisfies

$$
\begin{gathered}
\bar{L}_{\varepsilon}\left(\bar{u}-u^{h}\right)=\bar{f}-u_{i}^{h} \bar{L}_{\varepsilon} \phi_{i}-u_{i+1}^{h} \bar{L}_{\varepsilon} \phi_{i+1} \quad \text { on }\left(x_{i}, x_{i+1}\right), \\
\left(\bar{u}-u^{h}\right)\left(x_{i}\right)=\left(\bar{u}-u^{h}\right)\left(x_{i+1}\right)=0 .
\end{gathered}
$$

The local Green's function, $\bar{g}_{i}(x, y ; \varepsilon)$, for this constant-coefficient problem can be constructed explicitly and shown to satisfy

$$
\sup \left\{\left|\bar{g}_{i}(x, y ; \varepsilon)\right|, \varepsilon\left|\frac{\partial \bar{g}_{i}}{\partial x}(x, y ; \varepsilon)\right|: x_{i}<x, y<x_{i+1}, 0<\varepsilon \leq \varepsilon_{0}\right\} \leq c_{1}
$$

for some absolute constant $c_{1}$. It follows that

$$
\begin{aligned}
\left\|\bar{u}-u^{h} \mid\right\|_{\varepsilon,\left(x_{i}, x_{i+1}\right)} & \leq c_{1}\left\{\int_{x_{i}}^{x_{i+1}}|\bar{f}|+\left\|u^{h}\right\|_{\infty} \int_{x_{i}}^{x_{i+1}}\left(\left|\bar{L}_{\varepsilon} \phi_{i}\right|+\left|\bar{L}_{\varepsilon} \phi_{i+1}\right|\right)\right\} \\
& \leq c_{2} \cdot h, \quad 0<\varepsilon \leq \varepsilon_{0}, i=0, \ldots, n-1 .
\end{aligned}
$$

Our result now follows from the triangle inequality

$$
\left\|\left.\left|u-u^{h}\right|\right|_{\varepsilon} \leq\right\||| u-\bar{u}\left\|_{\varepsilon}+\right\|\left|\bar{u}-u^{h}\right| \|_{\varepsilon} .
$$

We obtain as corollaries of the above generalizations of results in [16, Section 6].

COROLlaRY 2.3. The following trial functions yield globally $\|\cdot\| \|_{\varepsilon}$-uniform $\mathscr{O}(h)$ schemes in the Petrov-Galerkin approximation (2.2) to (1.1):

(a) $\quad-\varepsilon \phi_{i}^{\prime \prime}+\bar{a} \phi_{i}^{\prime}+\bar{b} \phi_{i}=0 \quad$ on $\left(x_{i-1}, x_{i}\right) \cup\left(x_{i}, x_{i+1}\right)$,

$$
\phi_{i}\left(x_{i-1}\right)=\phi_{i}\left(x_{i+1}\right)=0, \quad \phi_{i}\left(x_{i}\right)=1 \text {; }
$$

(b) $\quad-\varepsilon \phi_{i}^{\prime \prime}+\tilde{a} \phi_{i}^{\prime}=0$ on $\left(x_{i-1}, x_{i}\right) \cup\left(x_{i}, x_{i+1}\right)$,

$$
\phi_{i}\left(x_{i-1}\right)=\phi_{i}\left(x_{i+1}\right)=0, \quad \phi_{i}\left(x_{i}\right)=1,
$$

for any $\tilde{a}=\bar{a}+\mathscr{O}(h)$.

Proof. Part (a) follows directly from Theorem 2.2, since $\bar{L}_{\varepsilon} \phi_{i}=0$ in each subinterval by construction. To establish the validity of the conclusion for the function in (b), note that

$$
\bar{L}_{\varepsilon} \phi_{i}=-\varepsilon \phi_{i}^{\prime \prime}+\bar{a} \phi_{i}^{\prime}+\bar{b} \phi_{i}=(\bar{a}-\tilde{a}) \phi_{i}^{\prime}+\bar{b} \phi_{i}
$$

implies

$$
\begin{aligned}
\int_{x_{i}}^{x_{i+1}}\left|\bar{L}_{\varepsilon} \phi_{i}\right| & \leq\|\bar{a}-\tilde{a}\|_{\infty} \cdot \int_{x_{i}}^{x_{i+1}}\left|\phi_{i}^{\prime}\right|+\|b\|_{\infty} \int_{x_{i}}^{x_{i+1}}\left|\phi_{i}\right| \\
& \leq\|\bar{a}-\tilde{a}\|_{\infty} \cdot \mathscr{O}(1)+\|b\|_{\infty} \cdot \mathscr{O}(h)=\mathscr{O}(h),
\end{aligned}
$$

and similarly for $\int_{x_{i}}^{x_{i+1}}\left|\bar{L}_{\varepsilon} \phi_{i+1}\right|$. 
In an analogous way, the local representation

$$
\left(\bar{u}-u^{h}\right)(x)=\int_{x_{i}}^{x_{i+1}} \bar{g}_{i}(x, y)\left(\bar{f}-\bar{L}_{\varepsilon} u^{h}\right)(y) d y
$$

can be used to establish uniform global accuracy with respect to the weaker Sobolev norm

$$
\|v \mid\|_{\varepsilon, 1}:=\max \left\{\|v\|_{1}, \varepsilon\left\|v^{\prime}\right\|_{1}\right\}
$$

for the trial space of piecewise linear "roof functions." These satisfy locally $\bar{L}_{\varepsilon} \phi_{i}=$ $\mathscr{O}(1 / h)$, and we get $\left\|e^{h}\right\| \|_{\varepsilon, 1}=\mathscr{O}(h)$ in this case.

Irrespective of the trial functions used, Theorem 2.2 guarantees that the nodal accuracy of the El-Mistikawy-Werle scheme is uniformly $\mathscr{O}(h)$. To get $\mathscr{O}\left(h^{2}\right)$ nodal accuracy requires a little more smoothness and more work. This can be attacked using local projections, as indicated for general superconvergence results by Douglas and Dupont in [2], or using the global Green's function, as in [12] and [16]. Both of these approaches are complicated by handling certain nonuniform behavior as $\varepsilon \rightarrow 0$, as is any analysis of these problems. Here we prefer to show how this higher rate of uniform convergence can be established in a few more strokes, using fairly traditional finite difference truncation/discretization error analysis.

3. Truncation Error. Associated with the finite difference approximation $(1.4) /(2.1)$ we have the local truncation operator, $T_{i}$, defined for smooth $v$ by

$$
\begin{aligned}
T_{i}[v]:= & L_{h, \varepsilon} v\left(x_{i}\right)-I_{h}\left(L_{\varepsilon} v\right)_{i} \\
= & \alpha_{i,-1} v\left(x_{i-1}\right)+\alpha_{i, 0} v\left(x_{i}\right)+\alpha_{i, 1} v\left(x_{i+1}\right) \\
& -\left\{\beta_{i,-1} L_{\varepsilon} v\left(x_{i-1}\right)+\beta_{i, 0} L_{\varepsilon} v\left(x_{i}\right)+\beta_{i, 1} L_{\varepsilon} v\left(x_{i+1}\right)\right\} \\
= & \frac{1}{h} \bar{B}_{\varepsilon}\left(v, \psi_{i}\right)-\frac{1}{h}\left(\overline{L_{\varepsilon} v}, \psi_{i}\right) \\
= & \frac{1}{h}\left[\bar{B}_{\varepsilon}\left(v, \psi_{i}\right)-B_{\varepsilon}\left(v, \psi_{1}\right)\right]+\frac{1}{h}\left(L_{\varepsilon} v-\overline{L_{\varepsilon} v}, \psi_{i}\right) \\
= & \frac{1}{h}\left((\bar{a}-a) v^{\prime}+\left(b^{\prime}-b\right) v+\left(L_{\varepsilon} v-\overline{L_{\varepsilon} v}\right), \psi_{i}\right) .
\end{aligned}
$$

The local truncation error, $\tau^{h}$, is then given by

$$
\tau_{i}^{h}:=T_{i}[u]=\frac{1}{h}\left((\bar{a}-a) u^{\prime}+(\bar{b}-b) u+(f-\bar{f}), \psi_{i}\right),
$$

where $u$ is the solution of (1.1). As a consequence of this definition, it follows that the discretization error, $e^{h}:=u-u^{h}$, satisfies

$$
L_{h, \varepsilon} e^{h}=\tau^{h} .
$$

A key to the analysis is the test function $\psi_{i}$, and we now establish a preliminary estimate concerning it. To simplify notation, all of this analysis is carried out on the generic subinterval $[-h, h]$ under an implicit transformation. The generic $\psi$ function is then defined by

$$
\begin{gathered}
-\varepsilon \psi^{\prime \prime}-\bar{a} \psi^{\prime}+\bar{b} \psi=0 \quad \text { on }(-h, 0) \cup(0, h), \\
\psi(-h)=\psi(h)=0, \quad \psi(0)=1,
\end{gathered}
$$

where $\bar{a}$ and $\bar{b}$ are piecewise constant functions defined in terms of the values of $a$ and $b$ at $-h, 0$, and $h$ as before. 
LEMMA 3.1. Under the assumptions (1.2), the local test function $\psi$ above satisfies

(a) $\psi \geq 0$

(b) $\max _{-h \leq x \leq h}|\psi(x)|, \int_{-h}^{h}\left|\psi^{\prime}\right|=\mathscr{O}(1)$,

(c) $\int_{-h}^{h} \psi \geq h / 2$,

(d) $\int_{-h}^{0}(x+h / 2) \psi+\int_{0}^{h}(x-h / 2) \psi=\mathscr{O}\left(h^{3}\right)$, uniformly in $0<\varepsilon \leq \varepsilon_{0}, 0<h \leq h_{0}$.

Proof. The nonnegativity of $\psi$ follows from the fact that $\bar{L}_{\varepsilon}$ cannot have any pairs of conjugate points in $[-h, h]$, as noted in the proof of Proposition 2.1. Now for $0<\varepsilon \leq \varepsilon_{1}, \psi$ is monotone increasing on $[-h, 0]$ and monotone decreasing on $[0, h]$, so that $0 \leq \psi \leq 1$ and

$$
\int_{-h}^{h}\left|\psi^{\prime}\right|=\int_{-h}^{0} \psi^{\prime}-\int_{0}^{h} \psi^{\prime}=2
$$

For $\varepsilon_{1} \leq \varepsilon \leq \varepsilon_{2}, \psi$ and $h \cdot \psi^{\prime}$ are uniformly bounded, and (b) is established.

Property (c) can be proved by observing that $\psi(x) \geq(x+h) / h$ on $-h \leq x \leq 0-$ a reflection of upwinding. And part (d) follows either by a direct (but messy) calculation or by observing that $\psi=\psi_{0}+\mathscr{O}(h)$, where $\psi_{0}$ satisfies

$$
\begin{gathered}
-\varepsilon \psi_{0}^{\prime \prime}-a_{0} \psi_{0}^{\prime}=0 \quad \text { on }(-h, 0) \cup(0, h), \\
\psi_{0}(-h)=\psi_{0}(h)=0, \quad \psi_{0}(0)=1
\end{gathered}
$$

and

$$
\int_{-h}^{0}\left(x+\frac{h}{2}\right) \psi_{0}+\int_{0}^{h}\left(x-\frac{h}{2}\right) \psi_{0}=0 .
$$

In our truncation error analysis, we will use, instead of local Taylor series type approximations, the following representation result for solutions of (1.1). This theorem is a consequence of a general representation result proved in [5] and refined somewhat in [4].

THEOREM 3.2. Under assumption (1.2) and the requirement $a, b, f \in \mathscr{W}^{2, \infty}$, the solution $u$ of (1.1) admits the representation

$$
u(x ; \varepsilon)=v(x ; \varepsilon)+w(x ; \varepsilon) \exp \left(-\frac{1}{\varepsilon} \int_{x}^{1} a\right)
$$

where $v$ and $w$ and their derivatives up to order 2 exist almost everywhere on $(0,1)$ and can be bounded uniformly in $0<\varepsilon \leq \varepsilon_{0}$.

We require one more preliminary estimate concerning the exponential layer type function.

LEMMA 3.3. Under assumption (1.2), the following inequality is valid for all $\varepsilon, h>0$ :

$$
\int_{-h}^{h}\left|\exp \left(-\frac{1}{\varepsilon} \int_{x}^{h} a\right)-\exp \left(-\frac{1}{\varepsilon} \int_{x}^{h} \bar{a}\right)\right| \leq \operatorname{ch} \min \{\varepsilon, h\},
$$

where $c$ depends only on a. 
Proof. From the Mean Value Theorem we get

$$
e^{-(1 / \varepsilon) \int_{x}^{h} a}-e^{-(1 / \varepsilon) \int_{x}^{h} \bar{a}}=e^{\alpha} \cdot\left(-\frac{1}{\varepsilon}\right) \cdot \int_{x}^{h}(a-\bar{a})
$$

for some $\alpha \in \operatorname{int}\left(-(1 / \varepsilon) \int_{x}^{h} a,-(1 / \varepsilon) \int_{x}^{h} \bar{a}\right)$. It follows that $\alpha \leq-(1 / \varepsilon) \underline{a}(h-x)$ and

$$
\begin{aligned}
\mid e^{-(1 / \varepsilon) \int_{x}^{h} a}-e^{-(1 / \varepsilon) \int_{x}^{h} \bar{a} \mid} & \leq e^{-(1 / \varepsilon) \underline{a}(h-x)} \cdot \frac{1}{\varepsilon}(h-x) \cdot \frac{1}{2}\left\|a^{\prime}\right\|_{\infty} h \\
& \leq\left(\frac{e^{-1}}{\underline{a}}\right) \cdot \frac{\left\|a^{\prime}\right\|_{\infty}}{2} \cdot h .
\end{aligned}
$$

The estimate in (3.1) when $h \leq \varepsilon$ follows directly from this.

To handle the case $\varepsilon \leq h$, we first note that

$$
\int_{-h}^{h}(h-x) e^{-(1 / \varepsilon) \underline{a}(h-x)}=-2 h \frac{\varepsilon}{\underline{a}} e^{-(2 / \varepsilon) \underline{a} h}+\left(\frac{\varepsilon}{\underline{a}}\right)^{2}\left(1-e^{-(2 / \varepsilon) \underline{a} h}\right) \leq\left(\frac{\varepsilon}{\underline{a}}\right)^{2} .
$$

It then follows from above that

$$
\int_{-h}^{h}\left|e^{-(1 / \varepsilon) \int_{x}^{h} a}-e^{-(1 / \varepsilon) \int_{x}^{h} \bar{a}}\right| \leq \frac{\left\|a^{\prime}\right\|_{\infty}}{2 \varepsilon} h \int_{-h}^{h}(h-x) e^{-(1 / \varepsilon) \underline{a}(h-x)} \leq \frac{\left\|a^{\prime}\right\|_{\infty}}{2 \underline{a}^{2}} \varepsilon h .
$$

We now establish our truncation error bound for the El-Mistikawy-Werle scheme.

THEOREM 3.4. Under assumption (1.2) and the requirement $a, b, f \in \mathscr{W}^{2, \infty}$, there exists a constant $c$ such that for $0<\varepsilon \leq \varepsilon_{0}, 0<h \leq h_{0}$, and $i=1, \ldots, n-1$,

$$
\begin{aligned}
\left|\tau_{i}^{h}\right| \leq \operatorname{ch}^{2}\{1+ & \frac{1}{\varepsilon} \exp \left(-\frac{1}{\varepsilon} \underline{a}\left(1-x_{i}\right)\right) \\
& \left.+\min \left\{\frac{1}{h}, \frac{1}{\varepsilon}\right\} \cdot \exp \left(-\frac{1}{\varepsilon} \underline{a}\left(1-x_{i+1}\right)\right)\right\} .
\end{aligned}
$$

Proof. Recall that

$$
\tau_{i}^{h}=\frac{1}{h}\left((\bar{a}-a) u^{\prime}+(\bar{b}-b) u+(f-\bar{f}), \psi_{i}\right)
$$

and

$$
u=v+w \exp \left(-\frac{1}{\varepsilon} \int_{x}^{1} a\right)
$$

where $v$ and $w$ have uniformly bounded derivatives up to second order. We first show that $\left(f-\bar{f}, \psi_{i}\right)=\mathscr{O}\left(h^{3}\right)$ :

$$
\begin{aligned}
(f-\bar{f}, \psi) & =\int_{-h}^{0}\left[f(x)-\frac{f(-h)+f(0)}{2}\right] \psi+\int_{0}^{h}\left[f(x)-\frac{f(0)+f(h)}{2}\right] \psi \\
& =\int_{-h}^{0}\left[f^{\prime}(0) \cdot\left(x+\frac{h}{2}\right)+\mathscr{O}\left(h^{2}\right)\right] \psi+\int_{0}^{h}\left[f^{\prime}(0) \cdot\left(x-\frac{h}{2}\right)+\mathscr{O}\left(h^{2}\right)\right] \psi \\
& =f^{\prime}(0) \cdot \mathscr{O}\left(h^{3}\right)+\mathscr{O}\left(h^{3}\right)=\mathscr{O}\left(h^{3}\right),
\end{aligned}
$$

using Lemma 3.1. In exactly the same way, the contributions $\left((\bar{a}-a) v^{\prime}, \psi_{i}\right)$ and $\left((\bar{b}-b) v, \psi_{i}\right)$ can be shown to be uniformly $\mathscr{O}\left(h^{3}\right)$.

The remaining terms of $\tau_{i}^{h}$ are

$$
\frac{1}{h}\left((\bar{a}-a)\left(w^{\prime}+\frac{a}{\varepsilon} w\right) e^{-(1 / \varepsilon) \int_{x}^{1} a}, \psi_{i}\right)+\frac{1}{h}\left((\bar{b}-b) w e^{-(1 / \varepsilon) \int_{x}^{1} a}, \psi_{i}\right)
$$


of which the most difficult is the part involving $a w / \varepsilon$. We write

$$
\begin{aligned}
& \left((\bar{a}-a) a w e^{-(1 / \varepsilon) \int_{x}^{1} a}, \psi_{i}\right) \\
& =e^{-(1 / \varepsilon) \int_{x_{i+1}}^{1} a}\left\{e^{-(1 / \varepsilon) \int_{x_{i}}^{x_{i}+1} \bar{a}}\left((\bar{a}-a) a w, e^{(1 / \varepsilon) \int_{x_{i}}^{x} \bar{a}} \psi_{i}\right)\right. \\
& \left.\quad+\left((\bar{a}-a) a w,\left(e^{-(1 / \varepsilon) \int_{x}^{x_{i}+1} a}-e^{-(1 / \varepsilon) \int_{x}^{x_{i}+1} \bar{a}}\right) \psi_{i}\right)\right\} .
\end{aligned}
$$

Now the function $\phi:=\exp \left(\frac{1}{\varepsilon} \int_{0}^{x} \bar{a}\right) \psi$ satisfies conditions that are locally adjoint to those of $\psi$ :

$$
\begin{gathered}
-\varepsilon \phi^{\prime \prime}+\bar{a} \phi^{\prime}+\bar{b} \phi=0 \quad \text { on }(-h, 0) \cup(0, h), \\
\phi(-h)+\phi(h)=0, \quad \phi(0)=1 .
\end{gathered}
$$

It satisfies a lemma like Lemma 3.1, and this can be used to prove that the term $\left((\bar{a}-a) a w, \exp \left(\frac{1}{\varepsilon} \int_{x_{i}}^{x} \bar{a}\right) \psi_{i}\right)$ above is uniformly $\mathscr{O}\left(h^{3}\right)$.

Lastly, we use Lemma 3.3 to appraise the final expression:

$$
\begin{aligned}
\mid((\bar{a}-a) a w, & \left.\left(e^{-(1 / \varepsilon) \int_{x}^{h} a}-e^{-(1 / \varepsilon) \int_{x}^{h} \bar{a}}\right) \psi\right) \mid \\
& \leq c \cdot h \cdot \int_{-h}^{h}\left|e^{-(1 / \varepsilon) \int_{x}^{h} a}-e^{-(1 / \varepsilon) \int_{x}^{h} \bar{a}}\right| \\
& \leq c^{\prime} h^{2} \min \{\varepsilon, h\} .
\end{aligned}
$$

So, combining these estimates,

$$
\begin{aligned}
\frac{1}{h} \mid( & \left.(\bar{a}-a) \frac{a}{\varepsilon} w e^{-(1 / \varepsilon) \int_{x}^{1} a}, \psi_{i}\right) \mid \\
& \leq \frac{c}{\varepsilon h} e^{-(1 / \varepsilon) \int_{x_{i+1}}^{1} a}\left\{e^{-(1 / \varepsilon) \int_{x_{i}}^{x_{i+1}} \bar{a}} h^{3}+h^{2} \min \{\varepsilon, h\}\right\} \\
& \leq c\left\{\frac{h^{2}}{\varepsilon} e^{-(1 / \varepsilon) \underline{a}\left(1-x_{i}\right)}+\min \left\{h, \frac{h^{2}}{\varepsilon}\right\} e^{-(1 / \varepsilon) \underline{a}\left(1-x_{i+1}\right)}\right\} .
\end{aligned}
$$

The two remaining terms involving $w$ can be estimated similarly, and (3.2) results.

4. Discretization Error. The El-Mistikawy-Werle scheme possesses a discrete stability property that is analogous to the continuous stability property (1.3). This can be established using general results of Niederdrenk and Yserentant [11]. We first define some notation. Let $v^{h}$ be an arbitrary mesh function. Let $D$ denote the forward difference operator

$$
D v_{i}^{h}=\frac{v_{i+1}^{h}-v_{i}^{h}}{h} .
$$

Define the discrete 1 -norm, $\|\cdot\|_{h, 1}$, and $\infty$-norm, $\|\cdot\|_{h, \infty}$, by

$$
\left\|v^{h}\right\|_{h, 1}:=h \sum_{i=1}^{n-1}\left|v_{i}^{h}\right|, \quad\left\|v^{h}\right\|_{h, \infty}:=\max \left\{\left|v_{i}^{h}\right|: i=0, \ldots, n\right\},
$$

and

$$
\left\|D v^{h}\right\|_{h, \infty}:=\max \left\{\left|D v_{i}^{h}\right|: i=0, \ldots, n-1\right\}
$$


The discrete weighted Sobolev norm, $|\| \cdot|||_{h, \varepsilon}$, is then given by

$$
\left\|v^{h}\right\|_{h, \varepsilon}:=\max \left\{\left\|v^{h}\right\|_{h, \infty}, \varepsilon\left\|D v^{h}\right\|_{h, \infty}\right\}
$$

We have the following

THEOREM 4.1. Under assumption (1.2), there exists a constant $c$ such that the finite difference scheme (1.4)/(2.1) satisfies

$$
\left\|\left|v^{h}\right|\right\|_{h, \varepsilon} \leq c\left\{\left\|L_{h, \varepsilon} v^{h}\right\|_{h, 1}+\left|v_{0}^{h}\right|+\left|v_{n}^{h}\right|\right\}, \quad 0<\varepsilon \leq \varepsilon_{0}, 0<h \leq h_{0},
$$

for any mesh function $v^{h}$.

Proof. It follows from results in [11]--see [5] or [6] for a description of those results for problems like ours - that all we have to do here is verify that the coefficients of our scheme satisfy (for each $i$ ) the "Niederdrenk-Yserentant" conditions:

(a) $\left|\alpha_{i,-1}+\alpha_{i, 0}+\alpha_{i, 1}\right| \leq M<\infty$,

(b) $h\left(\alpha_{i, 1}-\alpha_{i,-1}\right) \geq m>0$,

(c) $\alpha_{i,-1} \leq-\varepsilon / h^{2} \leq \alpha_{i, 1} \leq 0$,

where $m$ and $M$ are some constants that do not depend on $\varepsilon, h$, or $i$. Under these conditions, uniform consistency (in the sense that $\|T[\phi]\|_{h, 1} \rightarrow 0$ as $h \rightarrow 0$ uniformly in $\varepsilon$ for $\phi$ in the null space of $L_{\varepsilon}$ ) implies strong uniform stability, (4.1).

We verify these conditions. To simplify notation, we again transform to the interval $[-h, h]$. We first note that

$$
\begin{aligned}
\left|\alpha_{-1}+\alpha_{0}+\alpha_{1}\right| & =\frac{1}{h}\left|\bar{B}_{\varepsilon}(1, \psi)\right| \\
& =\frac{1}{h}\left|\int_{-h}^{h} \bar{b} \psi\right| \leq 2\|b\|_{\infty}\|\psi\|_{\infty}<\infty
\end{aligned}
$$

and

$$
\begin{aligned}
h\left(\alpha_{1}-\alpha_{-1}\right) & =\frac{1}{h} \bar{B}_{\varepsilon}(x, \psi)=\frac{1}{h} \int_{-h}^{h}\left(\varepsilon \psi^{\prime}+\bar{a} \psi+\bar{b} x \psi\right) \\
& =\frac{1}{h} \int_{-h}^{h}(\bar{a}+\bar{b} x) \psi \geq \frac{1}{h}\left(\underline{a}-h\|b\|_{\infty}\right) \frac{h}{2} \geq \frac{1}{4} \underline{a}>0,
\end{aligned}
$$

for all $h$ sufficiently small. Here we have used the facts ((a) and (c) of Lemma 3.1) that $\psi \geq 0$ and $\int_{-h}^{h} \psi \geq h / 2$. These give us (a) and (b).

Next observe that by taking $\phi_{-1}$ and $\phi_{1}$ in (2.1) to be the piecewise linear "roof functions" satisfying

$$
\phi_{-1}(-h)=\phi_{1}(h)=1, \quad \phi_{-1}(0)=\phi_{-1}(h)=\phi_{1}(-h)=\phi_{1}(0)=0,
$$

we get

$$
\alpha_{-1}=\frac{1}{h} \bar{B}_{\varepsilon}\left(\phi_{-1}, \psi\right)=\frac{1}{h^{2}}\left\{-\varepsilon-\int_{-h}^{0}(\bar{a}+\bar{b} x) \psi\right\}
$$

and

$$
\alpha_{1}=\frac{1}{h} \bar{B}_{\varepsilon}\left(\phi_{1}, \psi\right)=\frac{1}{h^{2}}\left\{-\varepsilon+\int_{0}^{h}(\bar{a}+\bar{b} x) \psi\right\} .
$$

Since $\bar{a}(x) \geq \underline{a}>0$ and $\psi \geq 0$, it follows that the two integrals above are nonnegative for all $h$ sufficiently small, and $\alpha_{-1} \leq \varepsilon / h^{2} \leq \alpha_{1}$. The fact that $\alpha_{1} \leq 0$ 
can be seen, for $0<\varepsilon \leq \varepsilon_{1}$, from a direct examination of the formula for $\alpha_{1}$, and (c) is established for all $0<\varepsilon \leq \varepsilon_{1}$ and $h$ sufficiently small. We can extend the validity of (4.1) by continuity to the entire range, $0<\varepsilon \leq \varepsilon_{0}$ and $0<h \leq h_{0}$, since we already know from Section 2 that our problem is well posed throughout that range.

Combining our truncation and stability results, we can now establish our main uniform convergence result for the El-Mistikawy-Werle scheme.

THEOREM 4.2. Under assumption (1.2) and the requirement $a, b, f \in \mathscr{W}^{2, \infty}$, there exists a constant $c$ such that the discretization error, $e^{h}=u-u^{h}$, of the finite difference scheme $(1.4) /(2.1)$ satisfies

$$
\left\|e^{h}\right\|_{h, \varepsilon} \leq c h^{2}, \quad 0<\varepsilon \leq \varepsilon_{0}, 0<h \leq h_{0} .
$$

Proof. As previously observed, the discretization error satisfies

$$
L_{h, \varepsilon} e^{h}=\tau^{h}, \quad e_{0}^{h}=e_{n}^{h}=0 .
$$

It follows from Theorem 4.1 that

$$
\left\|\mid e^{h}\right\|\left\|_{h, \varepsilon} \leq c\right\| \tau^{h} \|_{h, 1}, \quad 0<\varepsilon \leq \varepsilon_{0}, 0<h \leq h_{0} .
$$

But our truncation error bound (3.2) gives us

$$
\begin{aligned}
\left\|\tau^{h}\right\|_{h, 1} \leq \operatorname{ch}^{2}\left\{\|1\|_{h, 1}\right. & +\frac{1}{\varepsilon}\left\|\exp \left(-\frac{1}{\varepsilon} \underline{a}\left(1-x_{i}\right)\right)\right\|_{h, 1} \\
& \left.+\min \left\{\frac{1}{\varepsilon}, \frac{1}{h}\right\}\left\|\exp \left(-\frac{1}{\varepsilon} \underline{a}\left(1-x_{i+1}\right)\right)\right\|_{h, 1}\right\} .
\end{aligned}
$$

Now, $\|1\|_{h, 1} \leq 1$, and $\left\|\exp \left(-\frac{1}{\varepsilon} \underline{a}\left(1-x_{i}\right)\right)\right\|_{h, 1} \leq \| \exp \left(-\frac{1}{\varepsilon} \underline{a}(1-x) \|_{1} \leq \varepsilon / \underline{a}\right.$, by interpreting the discrete 1-norm of the exponential as a rectangle rule subapproximation to the continuous 1-norm.

We estimate the remaining piece as follows:

$$
\begin{aligned}
\left\|e^{-(1 / \varepsilon) \underline{a}\left(1-x_{i+1}\right)}\right\|_{h, 1} & =h \sum_{i=1}^{n-1} e^{-(1 / \varepsilon) \underline{a}\left(1-x_{i+1}\right)} \\
& =h \sum_{i=2}^{n-1} e^{-(1 / \varepsilon) \underline{a}\left(1-x_{i}\right)}+h \\
& \leq\left\|e^{-(1 / \varepsilon) \underline{a}(1-x)}\right\|_{1}+h \leq \frac{\varepsilon}{\underline{a}}+h .
\end{aligned}
$$

Combining these observations, we get

$$
\left\|r^{h}\right\|_{h, 1} \leq c h^{2}\left\{1+\frac{1}{\underline{a}}+\left(\frac{1}{\underline{a}}+1\right)\right\},
$$

and our uniform $\mathscr{O}\left(h^{2}\right)$ bound follows.

5. Concluding Remarks. These tools, the representation result of [4] and [5], here Theorem 3.2, and the stability theory of [11], seem to be very useful for the analysis of these types of problems. They have been used to analyze another wellknown finite difference approximation, the Allen-Southwell scheme, in [6]. There, 
results are obtained that, as in the present paper, are more general than previous analyses.

In the family of exponentially fitted HODIE schemes for problem (1.1), discussed in [5], there is a three-point formula that is similar to the El-Mistikawy-Werle scheme. Both use samplings of the data at $x_{i-1}, x_{i}$, and $x_{i+1}$ to compute the difference coefficients and weights for the $i$ th node. The former scheme has a classical convergence (for fixed $\varepsilon$ ) of $\mathscr{O}\left(h^{4}\right)$ and is uniformly $\mathscr{O}\left(h^{2}\right)$; while the ElMistikawy-Werle scheme is classically and uniformly $\mathscr{O}\left(h^{2}\right)$. The global extensions of the schemes are very different, and it turns out to be more effective to analyze them separately, rather than to try to deduce convergence properties of one based on direct comparison to the other.

Department of Mathematics

Southern Methodist University

Dallas, Texas 75275

1. A. E. Berger, J. M. Solomon \& M. Ciment, "An analysis of a uniformly accurate difference method for a singular perturbation problem," Math. Comp., v. 37, 1981, pp. 79-94.

2. J. DOUGlaS, JR. \& T. DUPONT, "Some superconvergence results for Galerkin methods for the approximate solution of two-point boundary value problems," in Topics in Numerical Analysis, Proc. Royal Irish Academy Conf., 1972 (J. J. H. Miller, ed.), pp. 89-92.

3. T. M. El-MistikAWY \& M. J. WERLE, "Numerical method for boundary layers with blowing - the exponential box scheme," AIAA J., v. 16, 1978, pp. 749-751.

4. E. C. GARTLAND, JR., Strong Stability and a Representation Result for a Singular Perturbation Problem, Technical Report AMS 87-1, Dept. of Mathematics, Southern Methodist University, January, 1987.

5. E. C. GARTLAND, JR., "Uniform high-order difference schemes for a singularly perturbed two-point boundary value problem," Math. Comp., v. 48, 1987, pp. 551-564.

6. E. C. Gartland, JR., An Analysis of the Allen-Southwell Finite-Difference Scheme for a Model Singular Perturbation Problem, Technical Report AMS 87-2, Dept. of Mathematics, Southern Methodist University, April, 1987.

7. A. F. HEgarty, J. J. H. Miller \& E. O'Riordan, "Uniform second order difference schemes for singular perturbation problems," in Boundary and Interior Layers-Computational and Asymptotic Methods (J. J. H. Miller, ed.), Boole Press, Dublin, 1980, pp. 301-305.

8. T. KATo, Perturbation Theory for Linear Operators, 2nd ed., Springer-Verlag, Berlin, 1980.

9. S. H. LeVenthal, "An operator compact implicit method of exponential type," J. Comput. Phys., v. 46, 1982, pp. 138-165.

10. J. LORENZ, Stability and Consistency Analysis of Difference Methods for Singular Perturbation Problems, Proc. Conf. on Analytical and Numerical Approaches to Asymptotic Problems in Analysis, June 9-13, 1980, Univ. of Nijmegen, The Netherlands (O. Axelsson, L. Frank, and A. Van der Sluis, eds.), North-Holland, Amsterdam, 1981.

11. K. NiederdRENK \& H. YSERENTANT, "Die gleichmässige Stabilität singulär gestörter diskreter und kontinuierlicher Randwertprobleme," Numer. Math., v. 41, 1983, pp. 223-253.

12. E. O'RIORDAN \& M. STYNES, "An analysis of a superconvergence result for a singularly perturbed boundary value problem," Math. Comp., v. 46, 1986, pp. 81-92.

13. M. H. Protter \& H. P. Weinberger, Maximum Principles in Differential Equations, Prentice-Hall, Englewood Cliffs, N. J., 1967.

14. D. R. Smith, A Green Function for a Singularly Perturbed Dirichlet Problem, Technical Report, Dept. of Mathematics, University of California, San Diego, March, 1984.

15. D. R. SMith, Singular Perturbation Theory, Cambridge Univ. Press, Cambridge, 1985.

16. M. STYNES \& E. O'RIORDAN, "A finite element method for a singularly perturbed boundary value problem," Numer. Math., v. 50, 1986, pp. 1-15.

17. W. G. SZYMCZAK \& I. BABUSKKA, "Adaptivity and error estimation for the finite element method applied to convection diffusion problems," SIAM J. Numer. Anal., v. 21, 1984, pp. 910-954. 